\title{
THE KUNZE-STEIN PHENOMENON
}

\author{
BY MICHAEL COWLING
}

Communicated by Robert T. Seeley, September 30, 1976

\begin{abstract}
We show that, if $G$ is a connected semisimple Lie group with finite center, then $L^{p}(G) * L^{2}(G) \subseteq L^{2}(G)$ if $1 \leqslant p<2$.

The theorem announced in the abstract was proved by R. A. Kunze and
\end{abstract} E. M. Stein [4] for the case when $G$ is $\operatorname{SL}(2, \mathbf{R})$. Various authors have extended to various other groups $G$, but the methods used have been quite technical and hard to generalise. The difficulty is the expression of the analytic continuation of the principal series as uniformly bounded representations on a Hilbert space. We avoid this difficulty by treating isometric representations on mixed $L^{p}$-spaces. Here is an outline of our method. We suppose that $G$ has real rank $r$.

Let $\bar{N} M A N$ be a Bruhat decomposition of $G$, and $\alpha_{1}, \ldots, \alpha_{r}$ the associated simple positive roots. We denote by $\bar{N}_{j}$ the subgroup of $\bar{N}$ whose Lie algebra is the sum of the root spaces $\Re_{-\alpha}$, where

$$
\alpha=m_{j} \alpha_{j}+m_{j-1} \alpha_{j-1}+\cdots+m_{1} \alpha_{1},
$$

with $m_{j}>0$. By $\rho_{j}$ we denote the element of the dual of the Lie algebra of $A$, defined by the rule

$$
\rho_{j}(a)=-1 / 2 \operatorname{tr}\left[\left.\operatorname{ad}(a)\right|_{\mathscr{R}_{j}}\right],
$$

and by $\rho$ the sum of the $\rho_{j}$. The group $\bar{N}$ has a decomposition $\bar{N}=\bar{N}_{r} \cdots \bar{N}_{1}$. Almost all elements of $G$ have a Bruhat decomposition: we write

$$
g=\bar{N}(g) M(g) A(g) N(g) .
$$

The unitary class-one principal series can be realised on $L^{2}(\bar{N})$ by the formula

$$
\left[\pi_{z}(g) \xi\right](\bar{n})=\exp \left[-\left(\rho+z_{1} \rho_{1}+\cdots+z_{r} \rho_{r}\right) \log A\left(g^{-1} n\right)\right] \xi\left(\bar{N}\left(g^{-1} n\right)\right),
$$

where $z$ is a purely imaginary $r$-tuple. Allowing $z$ to be complex in the above formula, we obtain one "analytic continuation of the principal series" (see Stein [5]). Let $L^{\mathbf{p}}(\bar{N})$ be the space of functions on $\bar{N}$ such that the norm \|\|$_{\mathrm{p}}$ :

$$
\|\xi\|_{p}=\left[\int_{N_{r}} d n_{r} \cdots\left[\int_{N_{1}} d n_{1}\left|\xi\left(n_{r} \cdots n_{1}\right)\right|^{p_{1}}\right]^{p_{2} / p_{1}} \cdots\right]^{1 / p_{r}},
$$

AMS (MOS) subject classifications (1970). Primary 22E30, 22 E45, 43 A22.

Key words and phrases. Semisimple Lie group, convolution, analytic continuation of the principal series. 
is finite. Then we have the following theorem:

THEOREM 1. Suppose that $p_{j} \operatorname{Re}\left(z_{j}\right)=2-p_{j}$. Then the representation $\pi_{z}$ acts isometrically on $L^{\mathfrak{p}}(\bar{N})$.

According to C. S. Herz [3], to prove the convolution theorem $\left(L^{p}(G) *\right.$ $L^{2}(G) \subseteq L^{2}(G)$ ) it suffices to show that, for $\xi$ and $\eta$ in $L^{2}(\bar{N})$, the function $g \longmapsto\left\langle\pi_{0}(g) \xi, \eta\right\rangle$ belongs to $L^{p^{\prime}}(G)$, or, equivalently, that

$$
\left\langle\pi_{0}(u) \xi, \eta\right\rangle \mid \leqslant C\|u\|_{p}, \quad u \in L^{1} \cap L^{p}(G) .
$$

We fix $\xi$ and $\eta$ in $L^{2}(\bar{N})$ of norm one. For $z$ in the tube $T$ of $r$-tuples with $\left|\operatorname{Re}\left(z_{j}\right)\right| \leqslant 1$, we define $\xi_{z}$ by the formulae:

and if $j>1$,

$$
\xi_{z}(\bar{n})=\xi(\bar{n}) \prod_{1}^{r} \xi_{j}(\bar{n}) \quad \text { where } \quad \xi_{1}(\bar{n})=|\xi(\bar{n})|^{z_{1}}
$$

$$
\xi_{j}(\bar{n})=\left[\int_{\bar{N}_{j}} d \bar{n}_{j} \cdots \int_{\bar{N}_{1}} d \bar{n}_{1}\left|\xi\left(\bar{n} \bar{n}_{j} \cdots \bar{n}_{1}\right)\right|^{2}\right]^{\left(z_{j}-z_{j-1}\right) / 2} ;
$$

$\eta_{z}$ is defined similarly, but $\bar{\eta}$ replaces $\xi$ and $-z_{j}$ replaces $z_{j}$. With these definitions, $\xi_{z}$ has norm one in $L^{\mathbf{p}}(\bar{N})$ and $\eta_{z}$ has norm one in the conjugate space. Thus, for $u$ in $L^{1}(G)$ and $z$ in $T$ we have the estimate

$$
\left|\pi_{z}(u) \xi_{z}, \eta_{z}\right\rangle \mid \leqslant\left\|\pi_{z}(u) \xi_{z}\right\|_{p}\left\|\eta_{z}\right\|_{p} \leqslant\|u\|_{1}\left\|\xi_{z}\right\|_{p}\left\|\eta_{z}\right\|_{p}=\|u\|_{1} .
$$

From the Plancherel formula for the class-one principal series (Harish-Chandra [1], [2] and G. Warner [6, Chapter 9]) we obtain the estimate

$$
\begin{aligned}
& {\left[\int_{\mathrm{R}} r y\left|C(y)^{-1}\left\langle\pi_{i y}(u) \xi_{i y}, \eta_{i y}\right\rangle\right|^{2}\right]^{1 / 2}} \\
& \leqslant\left[\int_{\mathrm{R}^{r}} d y\left|C(y)^{-1}\left\|\pi_{i y}(u)\right\|_{H S}\right|^{2}\right]^{1 / 2} \leqslant\|u\|_{2}
\end{aligned}
$$

for $u$ in $L^{1} \cap L^{2}(G)$. From the known properties of the $C$-function, it follows that there are some nonzero linear functionals $H_{j}$ on $\mathbf{R}^{r}$ and a constant $C$ such that

$$
\left.\prod_{1}^{k} \varphi\left(i H_{j}(y)\right)|\leqslant C| C(y)\right|^{-1},
$$

where $\varphi(z)=z / 2-z$. We now write $P_{z}(u)$ instead of $\left\langle\pi_{z}(u) \xi_{z}, \eta_{z}\right\rangle$, and our estimates (1) and (2) yield

$$
\begin{gathered}
\left|P_{z}(u)\right| \leqslant\|u\|_{1}, \quad u \in L^{1}(G), \\
{\left[\int_{R^{r}} d y\left|\prod_{1}^{k} \varphi\left(i H_{j}(y)\right) P_{i y}(u)\right|^{2}\right]^{1 / 2} \leqslant C\|u\|_{2} .}
\end{gathered}
$$

We note that $P_{z}(u)$ depends analytically on $z$, by the construction of $\pi_{z}, \xi_{z}$, and $\eta_{z}$. The main theorem is proved by applying the following interpolation theorem, whose proof is a mild inductive variation of Kunze and Stein's Theorem 4 and Lemma 26. 
THEOREM 2. Suppose that $z \longmapsto P_{z}$ is a weak-star topology analytic map of $T$ into $L^{\infty}(G)$, and that the estimates (3) and (4) hold. Then, if $1 \leqslant p<2$,

$$
\left|P_{0}(u)\right| \leqslant C\left(C, p, H_{1}, \ldots, H_{k}\right)\|u\|_{p}, \quad u \in L^{1} \cap L^{p}(G) .
$$

\section{REFERENCES}

1. Harish-Chandra, Harmonic analysis on semisimple Lie groups, Bull. Amer. Math. Soc. 76 (1970), 529-551. MR 41 \#1933.

2. - On the theory of the Eisenstein integral, Conf. on Harmonic Analysis (College Park, Md., 1971), Lecture Notes in Math., vol. 266, Springer-Verlag, Berlin and New York, 1972, pp. 123-149. MR 51 \#6285.

3. C. S. Herz, Sur le phénomène de Kunze-Stein, C. R. Acad. Sci. Paris Sér. A. 270 (1970), A491-A493. MR 43 \#6741.

4. R. A. Kunze and E. M. Stein, Uniformly bounded representations and harmonic analysis on the $2 \times 2$ real unimodular group, Amer. J. Math. 82 (1960), 1-62. MR 29 \#1287.

5. E. M. Stein, Analytic continuation of group representations, Advances in Math. 4 (1970), 172-207. MR 41 \#8584.

6. G. Warner, Harmonic analysis on semi-simple Lie groups. II, Grundlehren math. Wiss., Band 189, Springer-Verlag, Berlin and New York, 1972.

ISTITUTO DI MATEMATICA, UNIVERSITA DI GENOVA, 16132 GENOVA, ITALIA 